\title{
Analysis of the cues values of reinforcing brain stimulation
}

\author{
M. BEYRA and Ph. DE WITTE \\ Laboratoire de Neurophysiologie, Faculté de Medecine U. C. L., B-1200, Belgium
}

\begin{abstract}
Rats with rewarding electrodes in the lateral posterior hypothalamus were trained to an avoidance paradigm. A brain stimulation delivered through the rewarding electrode is used as conditioned stimulus. Test was made for stimulus generalization, presenting to animals several substitute stimulus (SS). SS were composed modifying the values of the electrical parameters of stimulation. The strength of the reinforcing effect elicited by this stimulus was evaluated. A high correlation was observed between the strength of the reinforcing effect and the stimulus generalization gradient. It is suggested that rats perceive the strength of reinforcing effect elicited by rewarding brain stimulation.
\end{abstract}

Since the classical studies of Loucks (cited by Grossman, 1967) and the positive evidence reached from Giurgea's and Doty's work (see references in Doty, 1969; Giurgea, 1964) that direct neural stimulation of the brain is an adequate conditioned stimulus (CS), the effects of direct electrical stimulation of the brain used as CS have been studied in a wide variety of conditioning paradigms. One aspect of these studies concerns the possibility of stimulus generalization between or within the different dimensions composing the conditioned stimulus; for instance, the physical parameters of the stimulus or the anatomical locus of stimulation. As Doty (1969) pointed out, it is observed that stimulation at points other than the original one to which the animal has been trained is without effect in eliciting the conditioned response, except when the CS and the substitute stimulus (SS) lie within the same functional system. Thus Stutz (1968) and Stutz, Butcher, \& Rossi (1969) reported a process of stimulus generalization if both the CS and the substitute stimulus were delivered in different anatomical structure of the rewarding brain system. Moreover, Butcher and Stutz (1969) reported that appropriate training is necessary to obtain a discrimination task between two rewarding brain stimulations delivered at different loci of the reinforcing brain system. These observations lead this author to assume that the most salient perceptual feature of subcortical stimulation, serving as cue to the animal, is the presence or absence of rewarding effect (Stutz, 1969).

It is observed that rats during intracranial self-stimulation (ICSS) are highly sensitive to the strength of the reinforcing effect elicited by rewarding brain stimulation (Beyra, 1974). It appeared interesting to pursue this problem studying the sensibility of rats to

The authors are greatly indebted to Professor M. Meulders for continuous support and constructive criticism. We are also grateful to the staff members of the laboratory of neurophysiology for helpful discussion. We are pleased to thank Mrs. F. Langhendries, Miss F. Ameels, Mr. C. Hendrick, C. Schouppe, and C. Stoquart for technical assistance. perceive the intensity of the reinforcing effect elicited by rewarding brain stimulation. Specifically, an avoidance conditioning was established using rewarding electrical stimulation as CS. Tests were made for stimulus generalization to other parameters of the electrical stimulus eliciting reinforcing effects of similar and different intensities to the reinforcing effect elicited by the CS.

\section{METHOD}

\section{Subjects, Electrodes, and Histology}

The subjects were male albino rats of the Wistar strain, weighing approximately $300 \mathrm{~g}$ at the time of operation. Each rat was implanted with one monopolar nickel-chrome electrode $(.25 \mathrm{~mm})$ insulated except for the cross section of the tips. The region aimed was the lateral posterior hypothalamus. The indifferent electrode was placed $2 \mathrm{~mm}$ forward the bregma. The electrodes were implanted stereotaxically according to the following coordinates: A, $3.5 \mathrm{~mm}$ behind bregma; $\mathrm{L}, 1.2 \mathrm{~mm}$; H, $8.3 \mathrm{~mm}$ below the skull surface. After the operation, the animals were allowed to recover 1 week and were trained for self-stimulation.

Following termination of the experiment, subjects were sacrificed, and the brain was removed from the skull and put in Formalin solution (10\% Formalin in saline) for 10 days. The brains were then frozen, sectioned at 100 microns, and stained with cresyl violet. The loci of the electrode tips were localized.

\section{Experimental Procedure}

We formed an experimental group of eight rats exhibiting self-stimulation behavior. Rats were placed in a shuttlebox and trained to avoid a footshock. A rewarding brain stimulation is the CS. The values of the electrical parameters of the brain stimulus were those raising a strong self-stimulation, i.e., $.64 \mathrm{sec}$ train duration, $: 3 \mathrm{msec}$ pulse width, delivered at the frequency of $200 \mathrm{~Hz}$. The current intensity remains constantly at the ICSS threshold (it varied between $150-280$ microA from rat to rat). The footshock was delivered $1 \mathrm{sec}$ at the offset of CS. Animals were trained twice a day during 30-min sessions; each session was composed of 30 trials with an intertrial interval lasting between 30 to $90 \mathrm{sec}$. Approximately 60 sessions were necessary to obtain the avoidance behavior. The criterion of acquisition was set at $100 \%$ of avoidance response. Five rats reached this criterion.

The generalization test was conducted by randomly interposing the substitute stimulus at the rate of one 
Table 1A

Mean Percentage of Avoidance Response (R\%) Elicited by the Conditioned (CS) and Substitute Stimulus (SS)

\begin{tabular}{|c|c|c|c|c|c|c|c|c|c|c|c|c|c|c|c|c|c|c|c|c|}
\hline & \multicolumn{5}{|c|}{ DP } & \multicolumn{5}{|c|}{$\mathrm{DP} / \mathrm{F}$} & \multicolumn{5}{|c|}{$\mathrm{DP} / \mathrm{I}$} & \multicolumn{5}{|c|}{$\mathrm{DP} / \mathrm{DT}$} \\
\hline & $\begin{array}{c}\text { DT } \\
\text { sc }\end{array}$ & $\begin{array}{c}\text { DP } \\
\text { msc }\end{array}$ & $\begin{array}{c}\mathrm{F} \\
\mathrm{Hz}\end{array}$ & $\underset{\mu \mathrm{A}}{\mathrm{I}}$ & $\begin{array}{l}\mathbf{R} \\
\%\end{array}$ & $\begin{array}{l}\text { DT } \\
\text { sc }\end{array}$ & $\begin{array}{c}\mathrm{DP} \\
\mathrm{msc}\end{array}$ & $\begin{array}{c}\mathrm{F} \\
\mathrm{Hz}\end{array}$ & $\underset{\mu \mathrm{A}}{\mathrm{I}}$ & $\begin{array}{l}\mathbf{R} \\
\%\end{array}$ & $\begin{array}{l}\text { DT } \\
\text { sc }\end{array}$ & $\begin{array}{c}\mathrm{DP} \\
\mathrm{msc}\end{array}$ & $\begin{array}{c}\mathrm{F} \\
\mathrm{Hz}\end{array}$ & $\underset{\mu \mathrm{A}}{\mathrm{I}}$ & $\begin{array}{l}\mathbf{R} \\
\%\end{array}$ & $\begin{array}{l}\text { DT } \\
\text { sc }\end{array}$ & $\underset{\mathrm{msc}}{\mathrm{DP}}$ & $\begin{array}{c}\mathrm{F} \\
\mathrm{Hz}\end{array}$ & $\underset{\mu \mathrm{A}}{\mathrm{I}}$ & $\begin{array}{l}\mathbf{R} \\
\%\end{array}$ \\
\hline $\mathrm{CS}$ & 0,64 & 0,3 & 200 & $\mathrm{Th}$ & 100 & 0,64 & 0,3 & 200 & $\mathrm{Th}$ & & 0,64 & 0,3 & 200 & $\mathrm{Th}$ & & 0,64 & 0,3 & 200 & $\mathrm{Th}$ & \\
\hline SS & 0,64 & 0,2 & 200 & $\mathrm{Th}$ & 64 & 0,64 & 0,2 & 200 & $\mathrm{Th}$ & 61 & 0,64 & 0,2 & 200 & Th & 67 & 0,64 & 0,2 & 200 & Th & 70 \\
\hline SS & & & & & & 0,64 & 0,2 & 250 & $\mathrm{Th}$ & 70 & 0,64 & 0,2 & 200 & +10 & 100 & 1,28 & 0,2 & 200 & $\mathrm{Th}$ & 94 \\
\hline SS & & & & & & 0,64 & 0,2 & 300 & $\mathrm{Th}$ & 78 & & & & & & & & & & \\
\hline SS & 0,64 & 0,15 & 200 & $\mathrm{Th}$ & 42 & 0,64 & 0,15 & 200 & $\mathrm{Th}$ & 26 & 0,64 & 0,15 & 200 & $\mathrm{Th}$ & 30 & 0,64 & 0,15 & 200 & $\mathrm{Th}$ & 51 \\
\hline SS & & & & & & 0,64 & 0,15 & 300 & $\mathrm{Th}$ & 78 & 0,64 & 0,15 & 200 & +10 & 77 & 1,28 & 0,15 & 200 & $\mathrm{Th}$ & 93 \\
\hline SS & & & & & & & & & & & 0,64 & 0,15 & 200 & +20 & 100 & 2,56 & 0,15 & 200 & $\mathrm{Th}$ & 98 \\
\hline SS & 0,64 & 0,1 & 200 & $\mathrm{Th}$ & 20 & 0,64 & 0,1 & 200 & $\mathrm{Th}$ & 0 & 0,64 & 0,1 & 200 & $\mathrm{Th}$ & 33 & 0,64 & 0,1 & 200 & $\mathrm{Th}$ & 25 \\
\hline SS & & & & & & 0,64 & 0,1 & 600 & $\mathrm{Th}$ & 46 & 0,64 & 0,1 & 200 & +20 & 58 & 1,28 & 0,1 & 200 & $\mathrm{Th}$ & 86 \\
\hline SS & & & & & & 0,64 & 0,1 & 800 & $\mathrm{Th}$ & 73 & 0,64 & 0,1 & 200 & +40 & 68 & 2,56 & 0,1 & 200 & $\mathrm{Th}$ & 83 \\
\hline SS & & & & & & 0,64 & 0,1 & 1000 & $\mathrm{Th}$ & 70 & 0,64 & 0,1 & 200 & +50 & 82 & 5 & 0,1 & 200 & $\mathrm{Th}$ & 41 \\
\hline SS & & & & & & & & & & & 0,64 & 0,1 & 200 & +70 & 96 & & & & & \\
\hline SS & 0,64 & 0,05 & 200 & $\mathrm{Th}$ & 3 & 0,64 & 0,05 & 200 & $\mathrm{Th}$ & 0 & 0,64 & 0,05 & 200 & $\mathrm{Th}$ & 5 & 0,64 & 0,05 & 200 & $\mathrm{Th}$ & 32 \\
\hline SS & & & & & & 0,64 & 0,05 & 800 & $\mathrm{Th}$ & 0 & 0,64 & 0,05 & 200 & +70 & 6 & 1,28 & 0,05 & 200 & $\mathrm{Th}$ & 37 \\
\hline SS & & & & & & 0,64 & 0,05 & 1000 & $\mathrm{Th}$ & 3 & 0,64 & 0,05 & 200 & +100 & 20 & 2,56 & 0,05 & 200 & $\mathrm{Th}$ & 60 \\
\hline SS & & & & & & 0,64 & 0,05 & 1500 & $\mathrm{Th}$ & 0 & & & & & & 5 & 0,05 & 200 & $\mathrm{Th}$ & 65 \\
\hline SS & & & & & & 0,64 & 0,05 & 2000 & $\mathrm{Th}$ & 0 & & & & & & & & & & \\
\hline SS & & & & & & 0,64 & 0,05 & 2500 & Th & 0 & & & & & & & & & & \\
\hline
\end{tabular}

Note. The SS and CS are tested on five different rats. The values of the electrical parameters forming the SS and the CS are represented: $D T=$ train duration in seconds, $D P=$ pulse width in milliseconds, $F=$ pulse frequency in cycles per second, and $I$ = current intensity in microamperes (Th represents that the current intensity remains constant at ICSS threshold).

Table 1B

Mean Percentage of Avoidance Response (R\%) Elicited by the Conditioned (CS) and Substitute Stimulus (SS)

\begin{tabular}{|c|c|c|c|c|c|c|c|c|c|c|c|c|c|c|c|c|c|c|c|c|}
\hline & \multicolumn{5}{|c|}{$\mathrm{F}$} & \multicolumn{5}{|c|}{$\mathrm{F} / \mathrm{DP}$} & \multicolumn{5}{|c|}{$\mathbf{F} / \mathbf{I}$} & \multicolumn{5}{|c|}{ F/DT } \\
\hline SS & 0,64 & 0,3 & 150 & Th & 87 & 0,64 & 0,3 & 150 & Th & 88 & 0,64 & 0,3 & 150 & $\mathrm{Th}$ & 85 & 0,64 & 0,3 & 150 & Th & 75 \\
\hline SS & & & & & & 0,64 & 0,4 & 150 & Th & 90 & 0,64 & 0,3 & 150 & +10 & 86 & 1,28 & 0,3 & 150 & Th & 100 \\
\hline SS & 0,64 & 0,3 & 100 & Th & 26 & 0,64 & 0,3 & 100 & $\mathrm{Th}$ & 17 & 0,64 & 0,3 & 100 & $\mathrm{Th}$ & 35 & 0,64 & 0,3 & 100 & Th & 11 \\
\hline SS & & & & & & 0,64 & 1 & 100 & Th & 100 & 0,64 & 0,3 & 100 & +40 & 82 & 5 & 0,3 & 100 & Th & 13 \\
\hline SS & 0,64 & 0,3 & 75 & Th & 7 & 0,64 & 0,3 & 75 & $\mathrm{Th}$ & 11 & 0,64 & 0,3 & 75 & Th & 12 & 0,64 & 0,3 & 75 & Th & 0 \\
\hline SS & & & & & & 0,64 & 0,8 & 75 & $\mathrm{Th}$ & 42 & 0,64 & 0,3 & 75 & +30 & 22 & 1,28 & 0,3 & 75 & Th & 28 \\
\hline SS & & & & & & 0,64 & 1 & 75 & Th & 91 & 0,64 & 0,3 & 75 & +50 & 42 & 2,56 & 0,3 & 75 & Th & 16 \\
\hline SS & & & & & & & & & & & 0,64 & 0,3 & 75 & +70 & 62 & 5 & 0,3 & 75 & Th & 13 \\
\hline SS & & & & & & & & & & & 0,64 & 0,3 & 75 & +100 & 97 & & & & & \\
\hline
\end{tabular}

Note. The SS and CS are, tested on five different rats. The values of the electrical parameters forming the SS and the CS are represented: $D T=$ train duration in seconds, $D P=$ pulse width in milliseconds, $F=$ pulse frequency in cycles per second, and $I=$ current intensity in microamperes, (Th represents that the current intensity remains constant at ICSS threshold).

presentation of the substitute stimulus on five or more presentations of the CS. Each substitute stimulus was presented 20 times to each rat. A total of 110 substitute stimuli were tested. A substitute stimulus consisted of brain stimulations presenting different values of the parameters of the electrical stimulation. The value received by the parameters of the electrical brain stimulus are represented on Table 1. The percentage of avoidance responses elicited by the substitute stimulus were used to estimate the stimulus generalization gradient.

The generalization test finished, the strength of the reinforcing effect elicited by the conditioned stimulus and a sample of 14 substitute stimuli eliciting different degrees of generalization were evaluated. This was done measuring the barpressing rate of rats for ICSS on a continuous reinforcement schedule. Each stimulus condition was presented to each rat five times, for 8-min periods, i.e., 40 min per subject and per brain stimulus conditions and $34 \mathrm{~h}$ of recorded performance for the sample. The barpressing rate elicited by substitute stimuli were expressed in percentage of barpressing rate elicited by the CS. The Bravais-Pearson correlation coefficient and the regression line between these two variables were then computed.

\section{RESULTS}

Table 1 represents the percentage of avoidance response elicited by the CS and SS. We observe that a decrease of the values received by one of the electrical parameters composing the CS (i.e., frequency of pulse, pulse width, current intensity, and train duration) is accompanied by a decrease in the percentage of avoidance responses (first column, Table. IA, B, C). This 
Table 1C

Mean Percentage of Avoidance Response (R \%) Elicited by the Conditioned (CS) and Substitute Stimulus (SS)

\begin{tabular}{|c|c|c|c|c|c|c|c|c|c|c|}
\hline & $\begin{array}{c}\text { DT } \\
\text { sc } \\
\end{array}$ & $\begin{array}{l}\mathrm{DP} \\
\mathrm{msc}\end{array}$ & $\begin{array}{r}\mathrm{F} \\
\mathrm{Hz}\end{array}$ & $\begin{array}{c}\mathbf{I} \\
\mu / \mathbf{A}\end{array}$ & $\begin{array}{l}\mathbf{R} \\
\% \\
\end{array}$ & $\begin{array}{c}\text { DT } \\
\text { sc } \\
\end{array}$ & $\begin{array}{c}\text { DP } \\
\text { msc }\end{array}$ & $\begin{array}{c}\mathrm{F} \\
\mathrm{Hz}\end{array}$ & $\begin{array}{c}\mathrm{I} \\
\mu / \mathrm{A}\end{array}$ & $\begin{array}{l}\mathbf{R} \\
\% \\
\end{array}$ \\
\hline & \multicolumn{5}{|c|}{ I } & \multicolumn{5}{|c|}{ I/DP } \\
\hline CS & 0,64 & 0,3 & 200 & Th & 100 & 0,64 & 0,3 & 200 & Th & \\
\hline SS & 0,64 & 0,3 & 200 & Th -40 & 62 & 0,64 & 0,3 & 200 & Th -40 & 82 \\
\hline SS & & & & & & 0,64 & 0,4 & 200 & Th-40 & 100 \\
\hline SS & 0,64 & 0,3 & 200 & Th-50 & 36 & 0,64 & 0,3 & 200 & Th-50 & 55 \\
\hline SS & & & & & & 0,64 & 0,4 & 200 & Th-50 & 85 \\
\hline SS & & & & & & 0,64 & 0,5 & 200 & Th-50 & 95 \\
\hline SS & 0,64 & 0,3 & 200 & Th- 60 & 30 & 0,64 & 0,3 & 200 & Th- 60 & 15 \\
\hline SS & & & & & & 0,64 & 0,5 & 200 & Th-60 & 72 \\
\hline SS & & & & & & 0,64 & 0,6 & 200 & Th-60 & 87 \\
\hline SS & 0,64 & 0,3 & 200 & Th-70 & 22 & 0,64 & 0,3 & 200 & Th-70 & 20 \\
\hline SS & & & & & & 0,64 & 0,5 & 200 & Th-70 & 40 \\
\hline SS & & & & & & 0,64 & 0,6 & 200 & Th-70 & 47 \\
\hline \multirow[t]{2}{*}{ SS } & & & & & & 0,64 & 0,7 & 200 & Th-70 & 72 \\
\hline & \multicolumn{5}{|c|}{$\mathrm{I} / \mathrm{F}$} & \multicolumn{5}{|c|}{ I/DT } \\
\hline $\mathrm{CS}$ & 0,64 & 0,3 & 200 & Th & & 0,64 & 0,3 & 200 & $\mathrm{Th}$ & \\
\hline SS & 0,64 & 0,3 & 200 & Th-40 & 65 & 0,64 & 0,3 & 200 & Th -40 & 65 \\
\hline SS & 0,64 & 0,3 & 250 & Th -40 & 90 & 1,28 & 0,3 & 200 & Th-40 & 92 \\
\hline SS & 0,64 & 0,3 & 300 & Th -40 & 100 & & & & & \\
\hline SS & 0,64 & 0,3 & 200 & Th-50 & 67 & 0,64 & 0,3 & 200 & Th-50 & 37 \\
\hline SS & 0,64 & 0,3 & 300 & Th-50 & 77 & 1,28 & 0,3 & 200 & Th-50 & 50 \\
\hline SS & 0,64 & 0,3 & 400 & Th-50 & 97 & 2,56 & 0,3 & 200 & Th-50 & 72 \\
\hline SS & 0,64 & 0,3 & 200 & Th -60 & 27 & 0,64 & 0,3 & 200 & Th-60 & 42 \\
\hline SS & 0,64 & 0,3 & 300 & Th -60 & 40 & 1,28 & 0,3 & 200 & Th- 60 & 57 \\
\hline SS & 0,64 & 0,3 & 400 & Th-60 & 62 & 2,56 & 0,3 & 200 & Th- 60 & 77 \\
\hline SS & 0,64 & 0,3 & 500 & Th-60 & 97 & & & & & \\
\hline SS & 0,64 & 0,3 & 200 & Th-70 & 17 & 0,64 & 0,3 & 200 & Th-70 & 22 \\
\hline SS & 0,64 & 0,3 & 400 & Th-70 & 22 & 2,56 & 0,3 & 200 & Th-70 & 70 \\
\hline SS & 0,64 & 0,3 & 500 & Th-70 & 22 & 5 & 0,3 & 200 & Th-70 & 60 \\
\hline SS & 0,64 & 0,3 & 600 & Th-70 & 25 & & & & & \\
\hline SS & 0,64 & 0,3 & 700 & Th-70 & 27 & & & & & \\
\hline
\end{tabular}

Note. The SS and CS are tested on five different rats. The values of the electrical parameters forming the SS and the CS are represented: $D T=$ train duration in seconds, $D P=$ pulse width in milliseconds, $F=$ pulse frequency in cycles per second, and $I=$ current intensity in microamperes (Th represents that the current intensity remains constant at ICSS threshold).

data represents a classical generalization gradient. It tends to support that the physical characteristics of the electrical stimulus have cues properties serving as CS.

In order to test if the physical characteristics of the electrical stimulus is the only dimension of the stimulus presenting cues properties, we have varied simultaneously the values of two parameters of the electrical stimulus. Table IA, B, and $C$ represent the values received in this eventuality by the electrical parameters of stimulation. These tables represent, also, the percentage of avoidance responses elicited by these stimulations. Figure 1 brings a visual illustration of this data. In this figure, as an example, the percentage of avoidance responses elicited by a decrease of pulse width accompanied by an increase of the current intensity (first line), a decrease of current intensity accompanied by an increase of pulse frequency (second line), and a decrease of pulse width accompanied by an increase of pulse frequency have been represented.

The data shows that the physical characteristics of the electrical stimulus is not the only dimension of the stimulus presenting cues properties. In fact, a substitute stimulus differing from the CS in the value of two parameters may evoke higher generalization than a substitute stimulus differing in the value of only one parameter. So, it appears logical to suppose that another dimension of the stimulus is the relevant cue serving as CS.

We assume that the relevant cue is the strength of the reinforcing effect elicited by brain stimulation. To test this hypothesis, we estimate the relation between the degree of generalization and the strength of the reinforcing effect elicited by a sample of substitute stimuli. Figure 2 represents the brain stimulus chosen to this purpose. We obtain a correlation coefficient (.84) and a regression that tend to support this hypothesis.

On the basis of this data, we assume that the strength of the reinforcing effect elicited by rewarding brain stimulation is a relevant cue. Consequently, it appears logical to conclude that rats perceive this characteristic of brain stimulation.

\section{DISCUSSION}

The data reported show that a stimulus generalization occurs when the electrical parameters of brain stimulation used as CS and the electrical parameter of brain stimulation used as substitute stimulus elicit a 

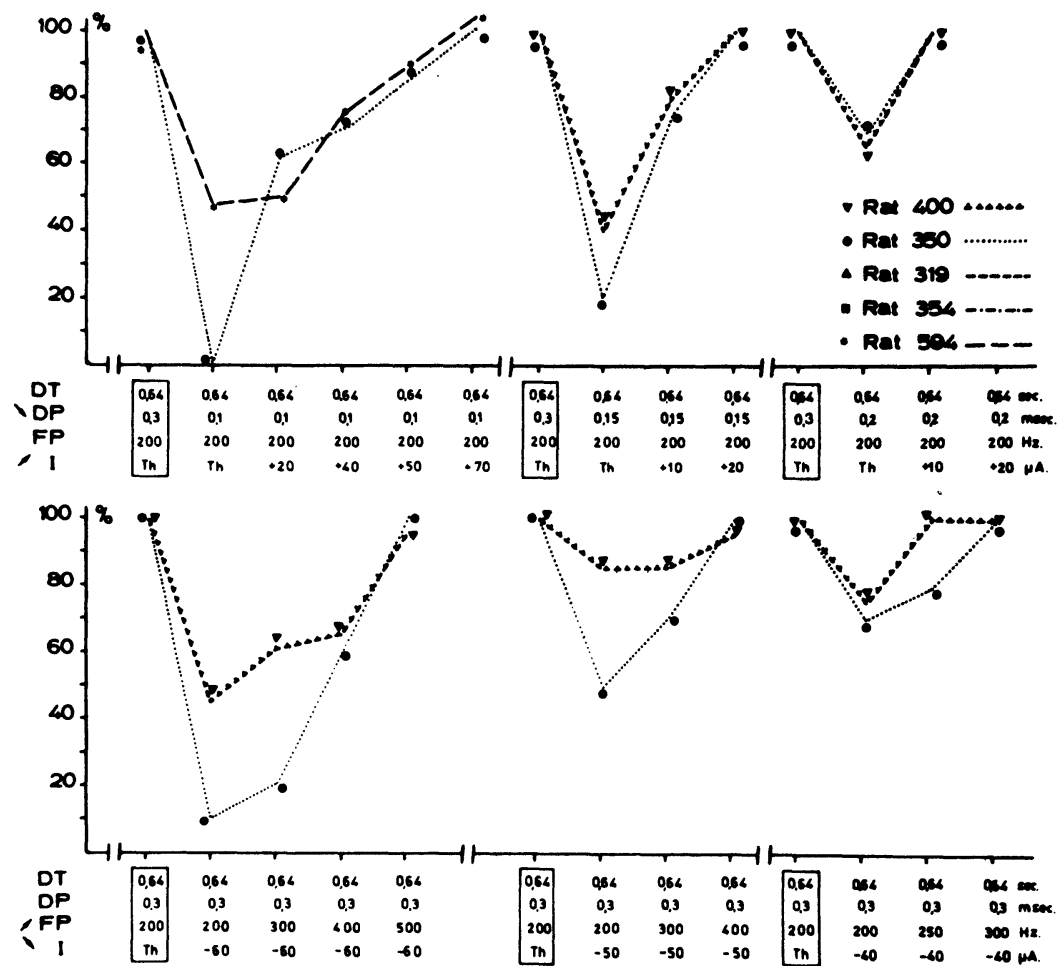

Figure 1. This figure presents the data. On the ordinate, the percentage of avoidance responses elicited is represented; the corresponding stimulus is represented on the abscissa. The CS is enclosed in a square. The arrows point out the parameters modified to form the SS. Observe that all the subjects tend to perform identically.

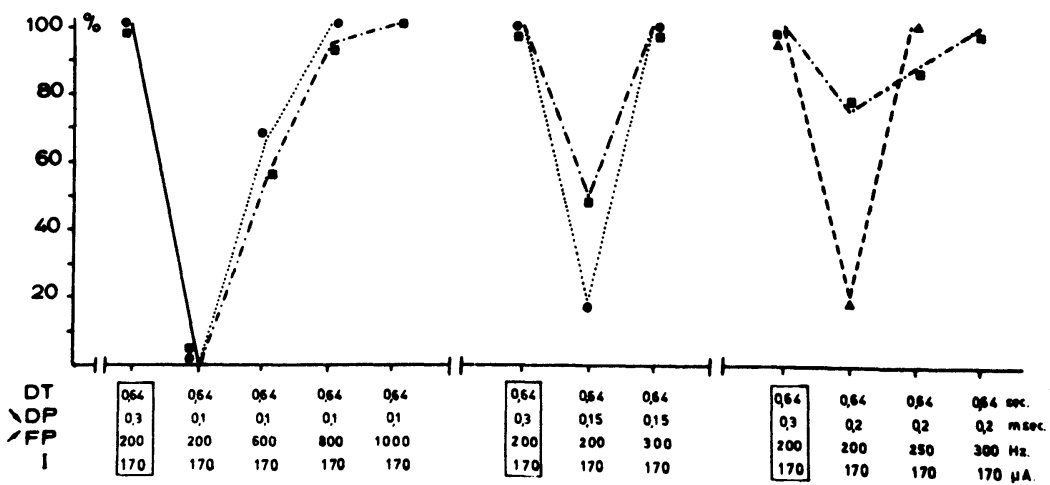

reinforcing effect of equal strength. A discrimination is observed when the brain stimulus used as substitute reinforcing effect elicited by the brain stimulus used as CS. It led us to assume, first, that the conditioned stimulus is composed of two dimensions, at least, i.e., the strength of the reinforcing effect and the physical characteristics of the stimulus. Secondly, we conclude that the strength of the reinforcing effect elicited by rewarding brain stimulation is a cue more relevant than the physical values of the parameters of electrical stimulation. On the ground of these assumptions, it is logical to assume that rats perceive the strength of the reinforcing effect elicited by brain stimulation.

Stutz's $(1968,1969)$ works show that stimulus generalization occurs when the CS and the substitute stimulus are applied to different points of the rewarding brain system. These data suggest that the valence of the affective nature elicited by brain stimulation (rewarding) is more pregnant than the locus of stimulation. The stimulus generalizations reported in this paper tend to show that the strength of the reinforcing effect is more pregnant than physical parameters of the electrical stimulus. Consequently, brain stimulation, as a CS, is a multidimensional stimulus. The degree of pregnance of these dimensions is too different for the dimensions to be similar.

These observations lead to the supposition that the learning strategy of the animal tend to stress one dimension of brain stimulation, in order to use it as CS. 

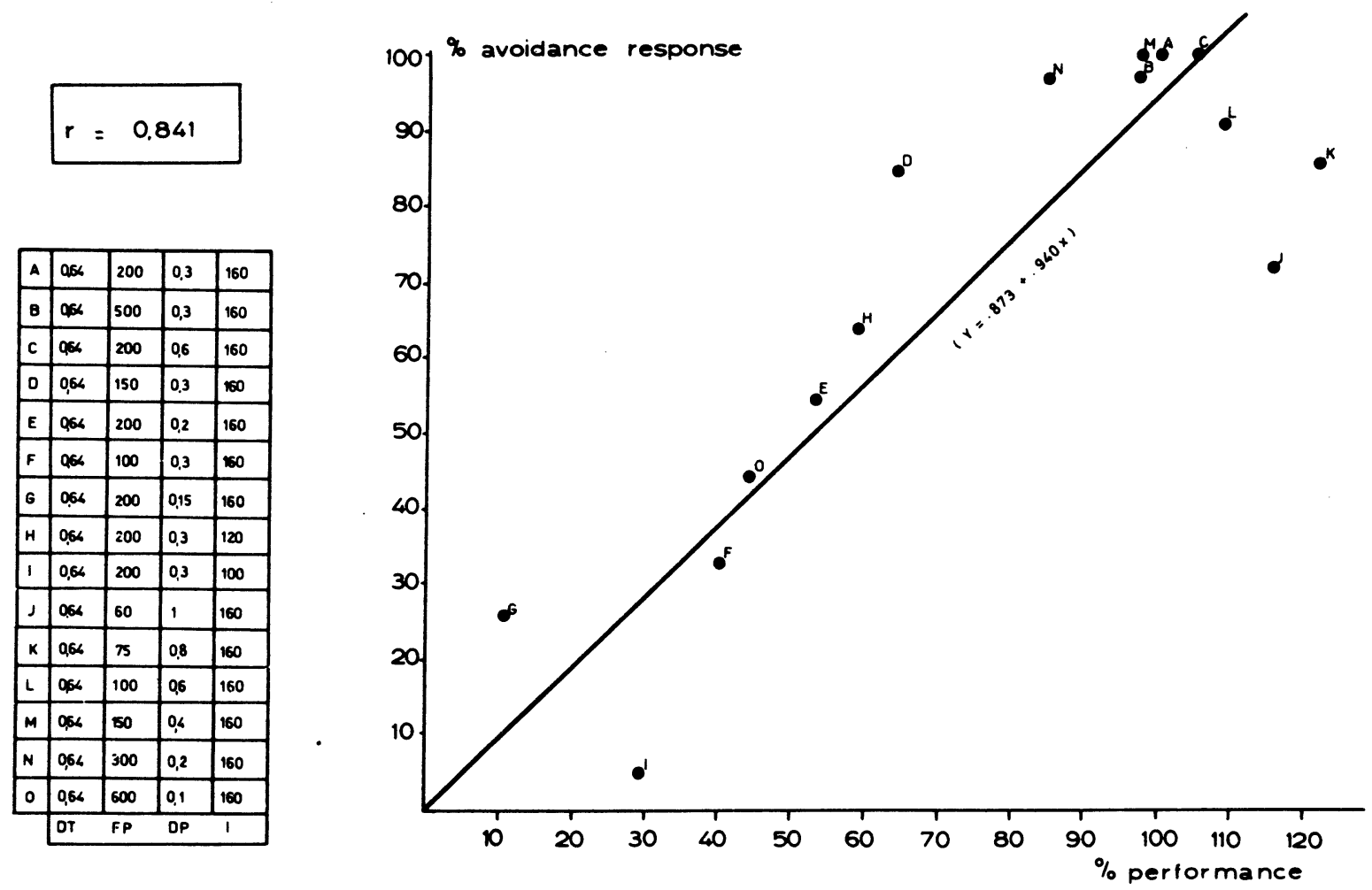

Figure 2. This figure represents the regression line. Avoidance response (on the ordinate) vs. strength of the reinforcing effect (on the abscissa) elicited by SS (coded by a letter and represented on the left panel). The CS is coded by the letter A. The ICSS barpressing rates elicited by the SS are expressed in percentage of the barpressing rate elicited by the CS. The Bravais-Pearson correlation ( $\mathbf{r}$ ) coefficient is also represented.

\section{REFERENCES}

BEYRA. M. The measurement of reinforcing brain stimulation. Brain Research, 1974, 70, 467-479.

Butcher. R. E., \& Stutz, R. M. Discriminability of rewarding subcortical brain shock. Physiology and Behavior, 1969. 4. 885-887.

Dоту. R. W. Electrical stimulation of the brain in behavioral content. Annual Review of Psychology, 1969, 28, 289-320.

Gurrgea, C. Neurophysiologie et conditionnement. Annales de la Societe Royale des Sciences Medicales et Naturelles. Bruxelles, 1969, 17, 58-88.
Grossman, A. A textbook of physiological psychology. New York and London: Wiley, 1967. Pp. 694-776.

STUTz. R. M. Stimulus generalization within the limbic system. Journal of Comparative and Physiological Psychology, 1968, 65, 79-82.

Stutz, R. M., Butcher, R. E., ANd Rossi, R. Stimulus properties of reinforcing brain shock. Science, 1969, 163, 1081-1082.

(Received for publication December 30, 1974; revision accepted May 8, 1975.) 